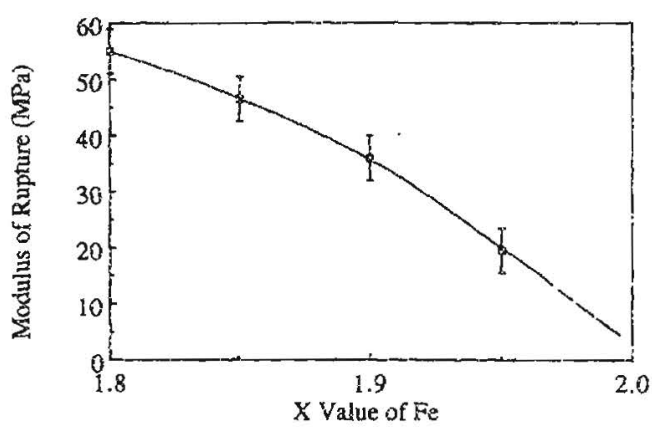

FIG. 2. Variation of modulus of ruprure with Fe stoichiometry in $\mathrm{RFe}_{\boldsymbol{x}}$.

of the convoluted nature of the dendritic morphology, ${ }^{4}$ it is highly probable that the rare-earth phase is aiso interconnected in the plane transverse to the growth direction. Consequently, the rare-earth phase is thought to be present as an interconnected skeleton network throughout the Terfenol-D matrix phase. The presence of a ductile skeleton network would be expected to enhance strength by retarding crack propazation throughout the brittle matrix. As $X$ is lowered from 2.0, the volume fraction of ductile rare-earth phase increases and the strength is improved. In general, the dark microconstituent in Fig. 1 is pure rare-earth metal when its transverse dimension is small but becomes a eutectic mixture of rare-earth metai and Terfenol-D phases when its dimension increases, such as at boundary trijunctions. This eutectic mixture becomes more dominant at the lower values of $X$, and a reduced ductility of the eutectic mixture over the pure rare-earth phase is probably responsible for the fallofi of the slope of the strength curve in Fig. 2 at the lowest $X$ values.

The magnetostrictive properties of Terfenol-D are only moderateiy reduced as $X$ decreases from 2.0 to $1.9{ }^{5}$ so that it is clearly advantageous to use values in the 1.9-1.95 range. The modulus of rupture values for $X$ in this range are similar to Eypical values for glass in accord with observations that Terfenol-D deforms only elastically and is fairly brittle. However, use of $X$ values in the 1.9-1.95 range produces a dramatic improvement in strength and elastic energy absorption, the fracture behavior is reproducible, and it is not as subject to the influence of surface finish as are some brittle materials.

The authors would ike to acknowledge the help of $L . K$. Reed in performing the mechanical tests, $J$. J. Holl for preparing the alloys, and $\mathrm{H}$. H. Baker in surface preparation of the samples. This work was done at the Ames Laboratory, which is operated for the United States Department of Energy by lowa State University, Contract No. W-7405-ENG. 82, supported by the Director of Energy Research, Office of Basic Energy Sciences.

'A. E. Clark, J. Culien, O. McMasters, and E. Cullen, Am. inst. Phys. Proc. $29,192(1976)$.

${ }^{2}$ A. E. Clark, J. P. Teters, and O. D. McMasters, J. Appl. Phys. 61, 3787 (1988).

${ }^{3}$ J. D. Verhoeven, A. J. Bevolo, D. T. Peterson, H. H. Baker, O. D. McMasters, and E. D. Gibson, Metallography 18, 277 (1985).

${ }^{4} J$. D. Verhoeven, E. D. Gibson, O. D. McMasters, and H. H. Baker, Metall.

Trans. 18A, 223 (1987).

${ }^{5}$ O. D. McMasters (unpublished).

\title{
Electron emission from glow-discharge cathode materials due to neon and argon ion bombardment
}

\author{
B. Szapiroa) and J. '. Rocca ${ }^{\text {b) }}$ \\ Electrical Engineering Deparment, Colorado State University, Fon Collins, Colorado 80523
}

(Received 14 November 1988; accepted for publication 4 January 1989)

\begin{abstract}
We have measured the electron yields of 7 glow-discharge cathode materials under bombardment by neon and argon ions with energies between 1 and $20 \mathrm{keV}$. The surfaces of the samples were conditioned by operating the materials as cold cathodes in a high-voltage glow discharge before the electron yield measurement. The materials studied are oxidized magnesium, oxidized aluminum, a molybdenum-aluminum oxide sintered composite, molybdenum, stainless steel, copper, and graphite. The dependence of electron yield on ion velocity was found to be approximately linear for all materials and gases, with the slopes being strongly thaterial dependent. The corresponding glow-discharge current intensities were observed to have a supralinear dependence or the electron yield. The results are relevant to the design and modeling of cold cathode high-voltage glow discharges.
\end{abstract}

The phenomenon of secondary electron emission from a solid surface under ion bombardment has been studied extensively, regardirig its importance in gas discharges, electron beam generation, piasma surface interactions, and ion

\footnotetext{
a) Post-Doctoral Fellow, University of Buenos Aires.

b) National Science Foundation Presidential Young Investigator.
}

beam current measurements. For example, in high-voitage cold cathode glow-discharge electron guns the electron yield of cathode surfaces is a key factor in determining the electron beam current dersities and generation efficiency. 'Careful measurements of the electron yieid have mostly been performed for atomically clean, fiat surfaces in ultrahigh vacuum conditions. ${ }^{2-7}$ However, these results usually do not 
apply for the operating conditions typical of high-voltage glow discharges, where sputtering effects, presence of oxide layers, and gas loading of the cold cathode surface influence the electron emissivity and thereby the discharge characteristics.

Previously, we measured the electron yields of cathode materials under helium ion bombardment for surface conditions similar to the ones present in cold cathode glow discharges. ${ }^{8}$ Here we report the electron yields for neon and argon ion bombardiment of sever cathode materials. The materials studied are oxidized magnesium, oxidized aluminum, a molybdenum-aluminum oxide sintered composite, molybdenum, stainless steel, copper, and graphite. In order to get electron yieid data applicable to typical high-voltage giowdischarge cathodes the surfaces of the samples were conditioned in situ by operating them as cathodes of a high-voltage glow discharge in a noble gas atmosphere of the same specie that the ion beam used for the yield measurements. For aluminum and magnesium a small amount of oxygen was added to the discharge to establish a stable oxide layer, as is frequentify done in high-volkage glow discharges to enhance the electron yield and decrease sputtering. ${ }^{1,2-i 1}$ The temporal evolution of the conditioning discharge is monitored until a steady-state value is reached. The discharge is then interrupted, the chamber is pumped down to $10^{-6}$ Torr and the sample is placed on axis with an ion gun to perform the electron yield measurements. The electron yieid is obtained by measuring the ion fiux impinging on the cathode and the total cathode current. After the yield measurement, the discharge is reestablished and the current is monitored to verify that the surface conditions remained unchanged. The experimental setup and procedure used to perform the measurements were described in greater detail in a previous publication. $^{8}$

The characteristics of the materials studied are similar in those used as cathodes in glow discharges: polycrystalline, commercial purity samples, machined, and poisshed and cleaned ultrasonically in methanol. The sintered composite was developed to be used as a high electron yield cathode material for dc electron beam glow discharges. ${ }^{1}$ The material composition is $40 \%$ aluminum oxide and $60 \%$ molybdenum by weight, containing particles approximately $10 \mu \mathrm{m}$ in diameter. The stainless steel is a 304 alloy.

The electron yields for neon and argon ion impact in the 1-20-keV energy range are presented in Figs. 1 and 2, respectively. At low ion energy (under $1 \mathrm{keV}$ ), where the potential electron emission contribution to the total yield becomes dominant, the curves should level of to a constant value; however, the energy spread of our ion beam did not allow us to accurately measure the electron yields in the low-energy range. As previously observed in the case of helium bombardment, ${ }^{8}$ oxidized magnesium and aluminum samples
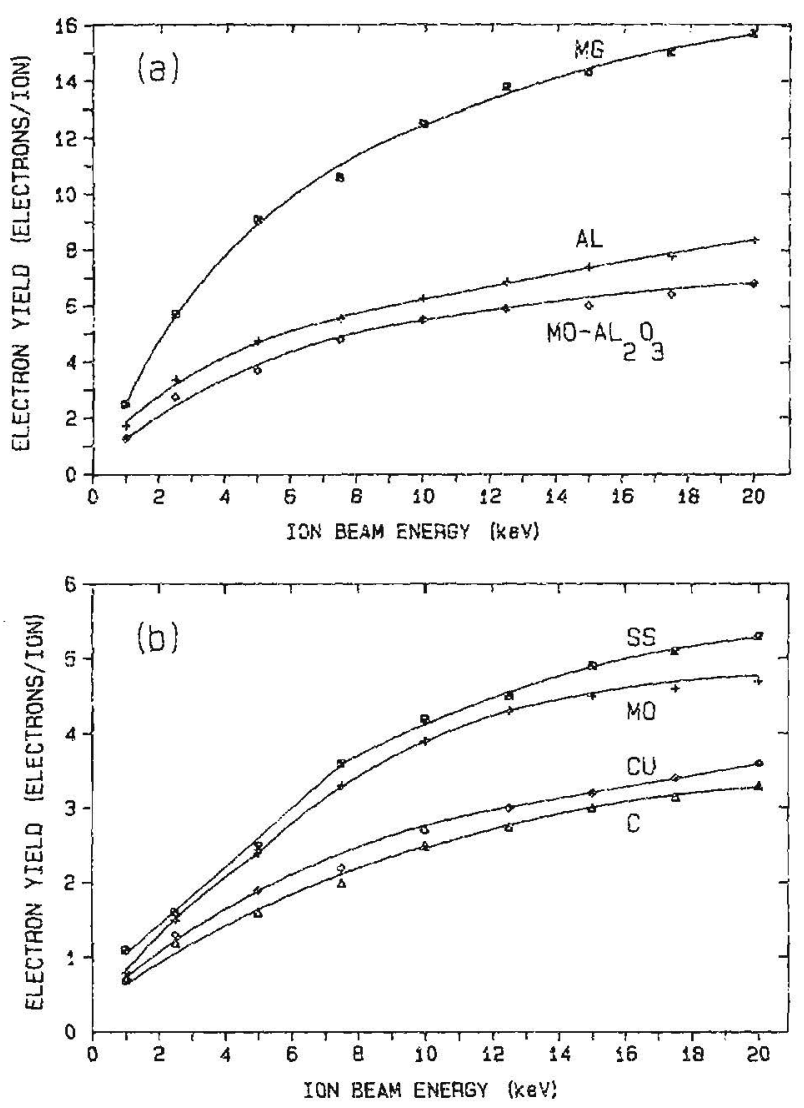

FIG. 1. Variation of the electron yield with steon ton beam energy. (a) Data for oxidized magnesium, oxidized aluminum, and a sintered composite Mo$\mathrm{Al}_{2} \mathrm{O}_{3}(60 \%-40 \%)$. (b) Data for stainless steel, molybdenum, copper, and graphite.
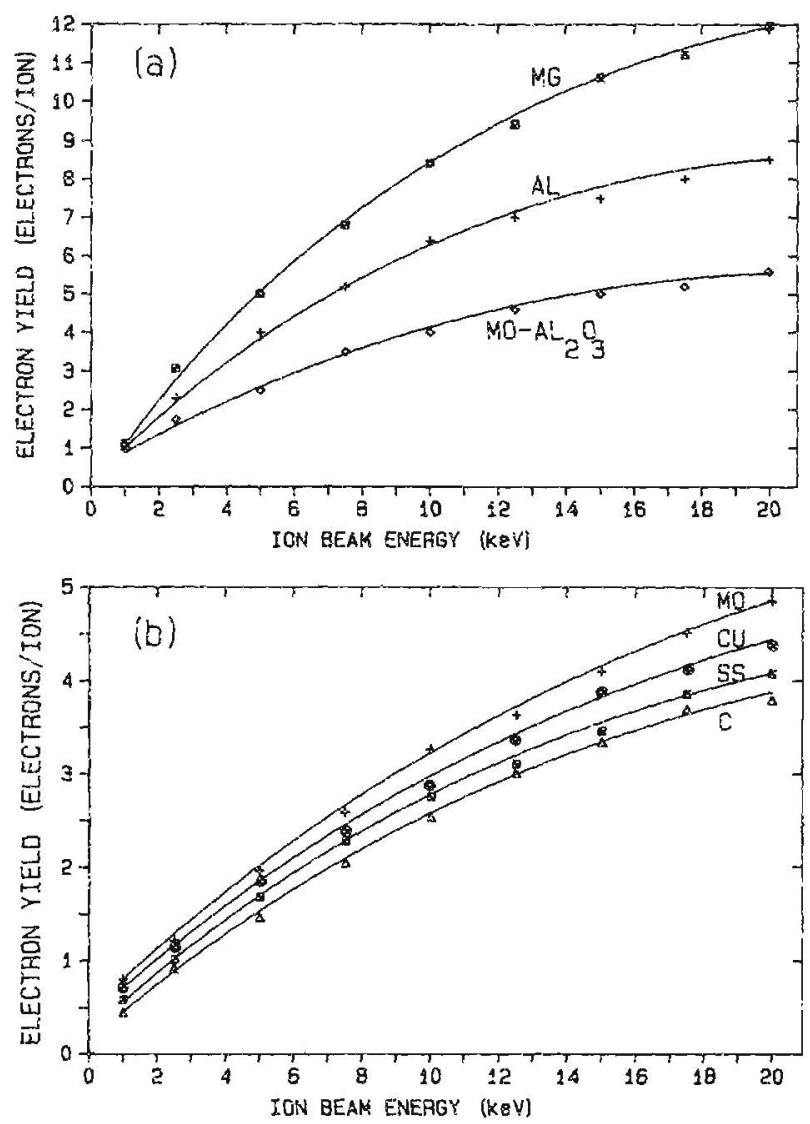

FIG. 2. Variation of the elcctron yiejd with argon ion beam energy. (a) Data for oxidized magnesium, oxidized aluminum, and a sintered composite $\mathrm{MO}-\mathrm{Al}, \mathrm{O},(60 \%-40 \%)$. (b) Data for molybdenum, copper, stainiess steel, and graphite. 
present the higher yields, followed by the sintered composite. The yields are a factor of 2-3 times higher than those measured under uitraclean conditions. "The increased yield in the discharged samples can be attributed to gas loading effects and increased surface roughness. Scanning electron microscope photogmaphs of the target surface show a significant increase in surface roughness due to discharge sputtering. It should be noticed that the resuits of Figs. 1 and 2 might be dependent on the discharge conditions. Nevertheless, measurements obtained after discharging the cathodes under significantly discharge currents (typically a threefold increase, with current densities in the order of $10 \mathrm{~mA} / \mathrm{cm}^{2}$ ) gave yield values differing by less than $10 \%$. Consequently, the results presented herein can be considered useful to describe conditions prevalent in many glow discharges, where the use of yieids measured under ultraclean conditions of flat, elemental surfaces would in general be inappropriate.

When the dat $a$ of Figs. 1 and 2 are plotted as a function of the ion velocity the depencience of the electron yields $Y$ on this parameter is found to be approximately linear for all cathode materials and ion species. This linear increase of the yield with ion velocity has been previousiy observed (for instance, see Refs. 12-14) in the energy range studied. The linear dependence with velocity arises from the proportionality between $Y$ and the electronic stopping power of the material $S_{c}\left(E_{i}\right)$ for an ion of initial energy $E_{i}$, and from the dependence $S_{e} \approx E_{i}^{1 / 2}$ in the energy range studied. ${ }^{14}$ For heavy ions, the contribution by recoiling target atoms should be included ${ }^{15} ;$ nevertheless, a simple semiempirical model developed by Baragiola, Alonso, and Oliva Florio, ${ }^{14}$ that neglects this effect, is observed to give a good description of the experimental data presented herein.

As expected, the slopes $d Y / d v$ are strongly dependent on the cathode materials ard ion species, ranging from $2.6 \times 10^{-8}$ electrons $/(\mathrm{cm} / \mathrm{s})$ for helium ion impact on graphite to $4.5 \times 10^{-7}$ electrons $/(\mathrm{cm} / \mathrm{s})$ for argon ion bombarcment on oxidized nagnesium. A comparison of our molybdenum data and those obtained for fiat surfaces in uitrahigh vacuum atmospheres (for instance, see Arifov, Rakhimov, and Khozinskii ${ }^{12}$ ) shows that our absolute yield values and slopes $d Y / d v$ are typically a factor of 2.5 higher, while the relative trends regarding the increase of the slopes with ion mass are similar, indicating that sputtering and gas loading effects produce an overall shift of the electron yields towards higher yalues.

Figures $3(a)$ and $3(b)$ illustrate the significance of the measured electron yields in determining the characteristics of glow discharges by relating the measured yields and the discharge current intensities delivered by the difierent materials. The data were obtained operating the samples as 1.2 $\mathrm{cm}$ in diameter glow-discharge cathodes by applying $2.5 \mathrm{kV}$ across the discharge and a $10-\mathrm{k} \Omega$ bailast resistor in a neon or argon atmosphere at a pressure of 0.2 and 0.1 Torr, respectively. These pressures were selected to allow in each case generation of a high-voltage sheath in front of the cathode, and thereby bombardment of the cathode by energetic ions. The cathodes are surrounded by ceramic tubes such that electron emission occurs only from the front surface. The electron yield values ised in these figures for the different
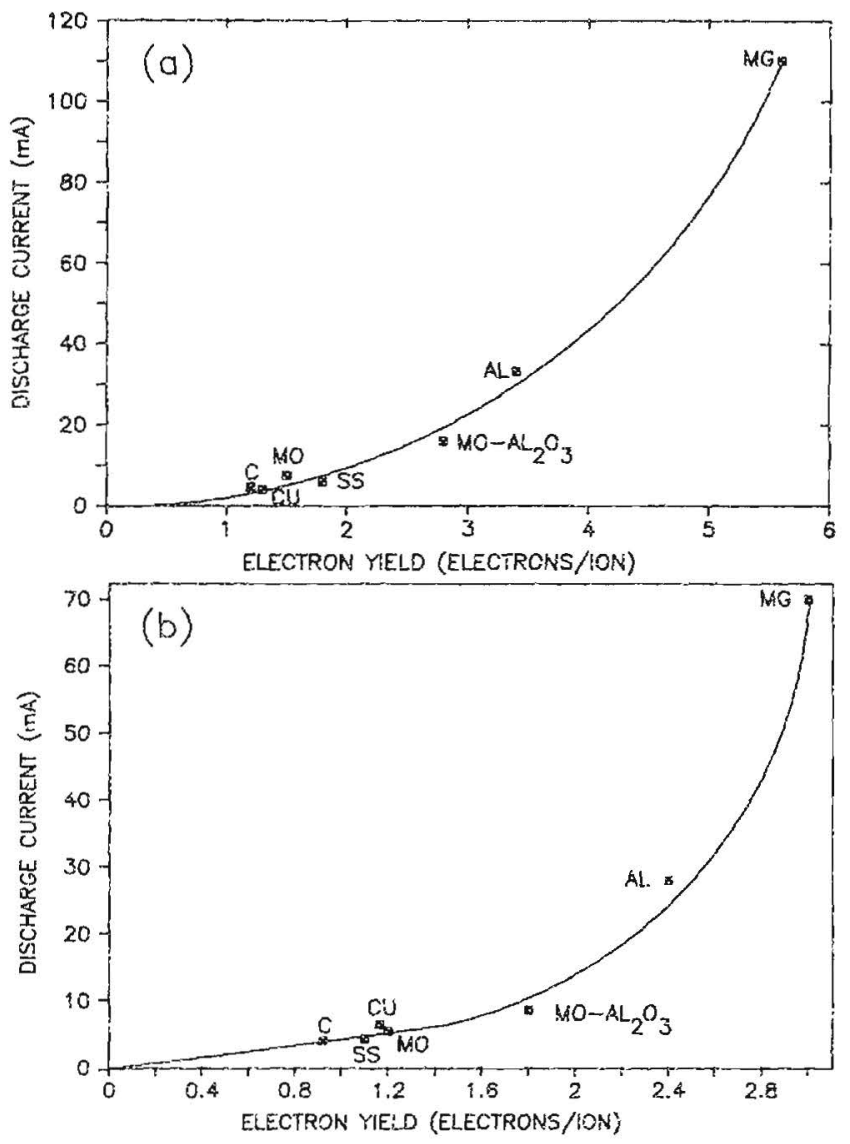

FlG. 3. Discharge current as a function of the electron yield for $2.5-\mathrm{keV}$ ions. (a) Data for neon. (b) Duka for argon. The cathodes are $1.2 \mathrm{~cm}$ in diameter. Discharge conditions are: a pressure of 0.2 -Torr neon or $0.1-$ Tor argon ( $10 \mathrm{~m}$ Torr of oxygen added in the aluminum and magnesium cathodes, $2.5-\mathrm{k} \forall$ voltage applied across the discharge and a $10-\mathrm{k} \Omega$ ballast resistor.

materials are those measured for a $2.5-\mathrm{keV}$ ion beam energy. A similar supralinear glow-discharge currení dependence on cathode electron yield was previously observed for helium ion bombardment and discussed in Ref. 8 . The sharp increase of the discharge current with the increase in cathode electron yield indicates the importance of selecting materials with high secondary electron emission coefficient, such as oxidized magnesium or oxidized aluminum, in the design of high-current cold cathode glow discharges.

This work was supported by Wright-Patterson AFB and AFOSR Grant No. 86-0096.

'3.J. Rucca, J. D. Meyer, M. R. Farrell, and G. J. Collins, J. Appl. Phys. 56, 790 (i984).

${ }^{2}$ M. Kaminski, Atumic and Lonic Impact Phenomena on Meial Surfaces (Springer, Berlin, 1965).

${ }^{3} \mathrm{G}$. Carter and J. S. Colligon, Ion Bombardment of Solids (Geinemann, London, :968\}.

${ }^{4}$ D. B. Medved and Y. E. Strausser, Adv. Electron. Eiectron Phys. 21, 101 (1965).

${ }^{5}$ U. A. Arifov, interaction of Atomic Particles With a Solid Surface (Consultants Bureau, New York, 1969).

${ }^{6}$ P. M. McCracken, Rep. Prog. Phys. 38, 241 (1975) 
${ }^{7}$ K. H. Krebs, Vacuum 33, 555 (1983).

${ }^{8}$ B. Szapiro, J. J. Rocea, and T. Prabhuram, Appl. Phys. Lett. 53, 358 (1988).

${ }^{9}$ R. E. Hurley and J. H. Hollyday, Vacuum 28, 453 (1978).

${ }^{10}$ B. Wernsman, H. F. Ranea Sandoval, J. J. Rocca, and H. Mancini, IEEE Trans. Plasma Sci. PS-14, 4, 518 (1986).

"H. F. Ranea Sandoval, N. Reesor, B. Szapiro, C. Murray, and J. J. Rocca,
IEEE Trans. Plasma Sci. PS-15, 4, 361 (1987),

"2 U. A. Arifov, R. R. Rakhimov, and O. Y. Khozinskii, Bull. Acad. Sci. (USSR) Phys. Ser. 26, 1422 (1963).

'YY. G. Tel'kovskii, Dokl. Akad. Nauk SSR 20, 1079 (1956).

${ }^{14}$ R. A. Baragiola, E. Y. Alonso, and A. Oliva Florio, Phys, Kev. B 19, 121 (1979).

15J. Sctiou, Phys. Rev. B 25, 2141 (1980).

\title{
High-power, difraction-limited-beam operation from interferometric, phase-locked arrays of AlGaAs/GaAs diode lasers
}

\author{
D. Botez, L. J. Mawst, P. Hayashida, and T. J. Roth \\ TRW Space \& Technology Group, One Space Park, M5/1065, Redondo Beach, California 90278
}

(Received 30 September 1988; accepted for publication 3 January, 1989)

\begin{abstract}
10/11-element interferometric phase-locked arrays with optimized facet coatings operate in array mode $l=8$, diffraction limited to $200 \mathrm{~mW}$ at 2.7 times thresholi, and in beam patterns 1.4 times the diffraction limit to $290 \mathrm{~mW}$ at 4.3 times threshoid. Unlike $Y$-junction-coupled arrays, the beam pattern quality is not sensitive to the facet(s) reflectivity value(s).

Transformation to a single-lobe pattern requires a simple phase-corrector coating or plate. The device beam pattern as a function of the array geometry is discussed.
\end{abstract}

Thompson and Whiteaway' have shown theoretically that high-order modes of evanescently coupled arrays are stable against gain spatial hole burning. We have reported ${ }^{2}$ that a $10 / 11$-element interferometric array, that selects operation in mode $L=8$, provides diffraction-limited-beam patterns to $\sim 85 \mathrm{~mW}$ and $\sim 3$ times threshold. Here we report on achieving $200 \mathrm{~mW}$ in a diffraction-limited-beam pattern, which, to the best of our knowledge, is the highest diffraction-limited power reported to date from 10-element phase-locked index-guided arrays. Furthermore, the results demorstrate that, unlike $Y$-junction coupled arrays, ${ }^{3}$ the use of antireflection coatings on the emitting facets of evanescent-wave coupled, interferometric arrays does not affect their beam pattern.

The fundamental mode of evanescently coupled arrays invariably broadens at output powers $\geqslant 40 \mathrm{~mW}^{4}$ or $1.5 \times$ threshold, ${ }^{4,5}$ due to gain spatial hole burning. ${ }^{1}$ Previously published work on evanescent-wave coupled arrays operating in a high-order mode, with optimized facet coatings, displays diffraction-limised beam operation to only 50 $\mathrm{mW}$ and $1.5 \times$ threshold, most probably due to weak discrimination against adjacent array modes. $Y$-junction coupled devices, while operating in stable fundamental-modelike patterns, have beamwidth $3.5-4$ times wider than the difraction limit. The overall interelement coupling is weak enough in $Y$-junction coupled devices, that their performance is highly sensitive to the feedback provided by the mirrors. Thus, when a low-reflectivity coating (3\%) is applied on one facet, the lobe beamwidth increases to six times the difiraction limit, ${ }^{3}$ due to weakened overall interelement coupling.
The interferometric arrays we report on have a similar structure to that reported on before ${ }^{2}$ : longitudinally the device is of the wide-waveguide interferometric (WWI) type; while the cross section is very similar to that shown in Fig. 1.

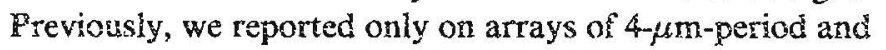
2- $\mu \mathrm{m}$-wide elements (Fig. 1), while this paper reports on devices of both 4- and 5- $\mu \mathrm{m}$ periods, and element widths varying in the $2-4 \mu \mathrm{m}$ range. $\mathrm{An} \mathrm{Al}_{0.10} \mathrm{Ga}_{0.90} \mathrm{As}$ antimeltback layer $(0.7 \mu \mathrm{m}$ thick) is initially grown by metalorganic vapor-phase epitaxy (MOVPE) above the substrate. The WWI pattern is etched through the antimeltback layer into the substrate, and then the rest of the structure is grown by liquid-phase epitaxy. The $p \cdot \mathrm{Al}_{0.30} \mathrm{Ga}_{0.70}$ As layer is $\sim 0.30$ $\mu \mathrm{m}$ thick in between elements. $42-\mu \mathrm{m}$-wide $\mathrm{SiO}_{2}$-defined metallic contact stripes are placed on the device $n$ side to provide current confinement. One facet is coated with a sixlayer dielectric stack $\left(\mathrm{Al}_{2} \mathrm{O}_{3} / \mathrm{Si}\right)$ refiector that provides $95 \%$ reflectivity at $\lambda=0.87 \mu \mathrm{m}$. The other facet is coated

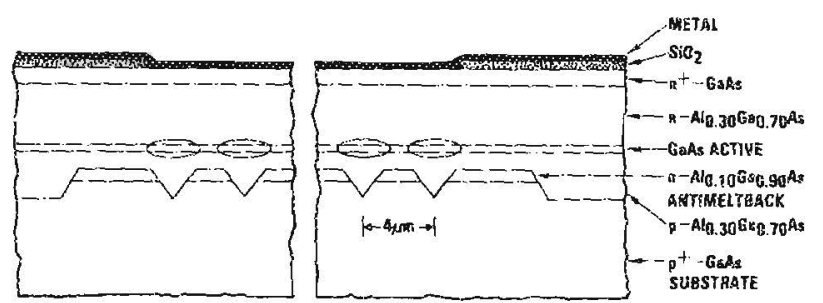

FIG. 1. Schematic respresentation of the emitring facet of the $10 / 1$-element array. Longitudinally the device has the wide-waveguide interferometric (WWI) geometry [see Fig. 1(a) in Ref. 2]. 